\title{
Stratégies d'exploitation du fourrage par les éleveurs de la zone sahélienne du Burkina Faso
}

\author{
André KIEMA $^{1 *}$, Issa SAWADOGO ${ }^{1}$, Tinrmegson OUÉDRAOGO ${ }^{1}$ et Aimé Joseph NIANOGO ${ }^{2,3}$ \\ ${ }^{1}$ Institut de l'Environnement et de Recherches Agricoles (INERA), 04 BP 8645 Ouagadougou 04, \\ Burkina Faso. \\ ${ }^{2}$ Institut du Développement Rural, Université Polytechnique de Bobo Dioulasso, 01 BP 1019 Bobo-Dioulasso, \\ Burkina Faso. \\ ${ }^{3}$ Union Mondiale pour la Conservation de la Nature (UICN), 01 BP. 3133 Ouagadougou 01, Burkina Faso. \\ *Auteur correspondant, E-mail : andre_kiema@yahoo.fr; inera.direction@fasonet.bf,
} Tél. : (+226) $50340270 / 50347112$

\section{RESUME}

Les caractéristiques de la collecte et de la conservation du fourrage ont été étudiées dans cinq villages du Sahel. L'objectif visé était d'identifier les principales stratégies utilisées par les paysans pour minimiser les problèmes de disponibilités alimentaires du fourrage en saison sèche. Des enquêtes à passage unique ont été ainsi conduites dans 205 exploitations d'agro pasteurs. Les résultats de l'étude révèlent que l'activité de production et de conservation du fourrage est largement pratiquée par les agropasteurs (plus de 88,2\%). La collecte concerne aussi bien les fourrages naturels que les résidus de récoltes. Les quantités totales de matières sèches stockées annuellement par exploitant sont de l'ordre de 2,043 tonnes, soit respectivement 13,5\% et $85,4 \%$ sous forme de fourrages naturels et de résidus de récoltes. Sur la base des ressources productives (nombre d'animaux) quatre classes de paysans ont été distinguées. En saison sèche, ces classes déploient des stratégies différentes en matière de gestion des stocks alimentaires. Les producteurs les moins nantis en ressources animales ont tendance à être des vendeurs nets de fourrages tandis que les plus nantis des acheteurs. Les principales contraintes aux activités d'exploitation du fourrage naturel sont surtout d'ordre économique mais aussi de gestion de l'espace.

(C) 2012 International Formulae Group. All rights reserved.

Mots clés: Fauche, résidus de récolte, fourrage naturel, saison sèche, éleveur, Burkina Faso.

\section{INTRODUCTION}

$\mathrm{Au}$ cours des dernières décennies, l'élevage du Burkina Faso a été marqué par un accroissement des effectifs du cheptel (bovins, ovins, caprins, camelins, asins, équins, etc.) (MRA, 2011). Cet accroissement est surtout lié à la maîtrise des grandes maladies animales, associée à l'explosion démographique, à l'amélioration des techniques d'élevage, etc.; ce qui a entraîné, naturellement, une augmentation des besoins en fourrage.

Si pendant les premiers moments, cette hausse continue des besoins alimentaires du bétail n'a pas constitué un problème compte tenu des disponibilités fourragères de l'époque, la situation a progressivement changé. En effet, l'extension rapide des surfaces cultivées (en relation avec la démographie galopante), les sécheresses 
répétées, l'érosion et les dégradations multiformes provoquées par les hommes et les animaux, ont fortement réduit les espaces pâturables.

L'action conjuguée de ces différents facteurs s'est traduite par une difficulté croissante d'alimentation des animaux et une importante fluctuation saisonnière des performances de productions animales. Pour pallier à cette situation, les éleveurs ont souvent opté pour la transhumance et quelquefois, la migration vers des zones écologiques plus favorables (Claude et al., 1991; Kiema, 2002; Boutrais, 2007 ; Sawadogo, 2012). Malheureusement, ces déplacements sont devenus de plus en plus difficiles car sources de nombreux conflits avec les populations des régions d'accueil.

Face à cette situation, de nombreux éleveurs tentent de trouver sur place les voies et moyens nécessaires pour nourrir leurs animaux. A ce titre, de nombreux efforts ont été déployés pour la production et la conservation du fourrage en saison sèche. Cet article identifie les principaux déterminants de cette production de façon à contribuer à minimiser les contraintes de disponibilité alimentaire.

\section{MATÉRIEL ET MÉTHODES}

\section{Présentation de la zone d'étude}

L'étude s'est déroulée dans cinq villages tous situés en région sahélienne du Burkina Faso: Yakouta, Lely, Arbinda, Tongomael et Pobé Mengao (Figure 1). Les villages ont été sélectionnés en fonction de leurs critères agro-écologiques pour la région. Ils s'étendent entre les latitudes $13^{\circ} 55^{\prime}$ et $14^{\circ} 05^{\prime}$ nord et les longitudes $0^{\circ} 00^{\prime}$ et $0^{\circ} 10^{\prime}$ ouest dans le secteur sahélien selon le découpage de Guinko (1984). Ils sont donc caractérisés par un climat de type sahélien ou sahélo soudanien suivant leur position géographique. La moyenne pluviométrique est située entre 400 à $600 \mathrm{~mm}$ pour la zone avec une période d'hivernage qui s'étale sur 3 à 4 mois (juin / juillet - septembre). La répartition des pluies connaît une grande variation interannuelle et inter-spatiale. Les sols sont essentiellement à textures sableuses sur les ensablements, argileuses sur les bas-fonds, argileuses, sableuses à limoneuses sur les glacis.

La végétation naturelle est dominée par des steppes qui se caractérisent essentiellement par des formations herbeuses ouvertes. Elle assure la transition entre les savanes soudaniennes à andropogonées et combrétacées situées plus au sud et la végétation désertique (Guinko, 1984).

Comme pour toutes les régions au Sahel, il existe une cohabitation entre plusieurs groupes ethniques: les Peul, les anciens captifs (Bella, Rimaïbé), les Mossi, etc.

L'agriculture et l'élevage sont les principales activités socio-économiques des habitants des villages. L'agriculture est essentiellement basée sur la culture du mil qui occupe les sols sableux et le sorgho installé sur les sols limono-argilo-sableux. Il existe une association de niébé et de mil ; l'arachide, le gombo et l'oseille sont faiblement cultivés. L'élevage est pratiqué par les agriculteurs essentiellement sédentaires qui possèdent du bétail (bovins, ovins, caprins). Les zones de pâture s'établissent sur les glacis. L'artisanat est une activité secondaire qui mobilise seulement quelques producteurs. Il est surtout dynamique en saison sèche avec les activités telles le tissage, la poterie, la vannerie, la cordonnerie, etc.

\section{Collecte de données}

Une phase de pré-enquête a été effectuée pour tester la fiche d'enquête afin de s'assurer que le questionnaire permettait d'obtenir toutes les informations recherchées. Le questionnaire standardisé après correction a été administré à 205 chefs d'exploitation entre décembre et février. Le taux d'échantillonnage a varié selon le village, entre 15 et $30 \%$ en fonction de la taille totale des exploitations.

Le questionnaire comporte cinq parties :

- La première partie permet de recueillir des informations sur les exploitations enquêtées 
(âge, taille du ménage, ethnie, scolarisation, activités principales, effectif du cheptel, etc.).

- La deuxième partie recueille les informations sur les espèces fauchées, les quantités stockées et l'évolution ces dernières années, de la pratique de l'activité. L'évaluation des quantités stockées a été faite à partir du dénombrement des bottes suivi de la pesée d'une dizaine pour le calcul des quantités totales stockées par espèces. En plus de cette évaluation, dix échantillons par site et par espèce ont été prélevés de façon aléatoire chez différents producteurs pour constituer un échantillon composite par site en vue de l'évaluation de la qualité des fourrages stockés. Les analyses bromatologiques ont consisté à l'estimation des teneurs en matière sèche (MS), matière organique (MO), l'azote total (MAT), la cellulose brute (CB). Les matières azotées digestibles (MAD) ont été calculées selon la formule de Demarquilly in Boudet (1991) et les unités fourragères selon la table de calcul des U.F. de Dijkstra (Boudet, 1991).

- La troisième partie traite de la gestion des ressources fourragères stockées (affectation des types de fourrage par catégorie d'animaux et en fonction des périodes).

- La quatrième partie aborde les aspects liés aux matériels utilisés (pour la collecte, le transport), les formations reçues en matière de production et gestion du fourrage, les périodes et temps consacrés à l'activité, les techniques de conditionnement et les infrastructures de stockage.

- La partie cinq du questionnaire traite de la réglementation de cette activité au niveau village (champs et pâturage naturel), les avantages et les inconvénients de cette activité, les difficultés rencontrées et les suggestions faites pour une amélioration.

Les informations ont d'abord été codifiées, puis des bases structurées ont été créées avant la saisie des données.

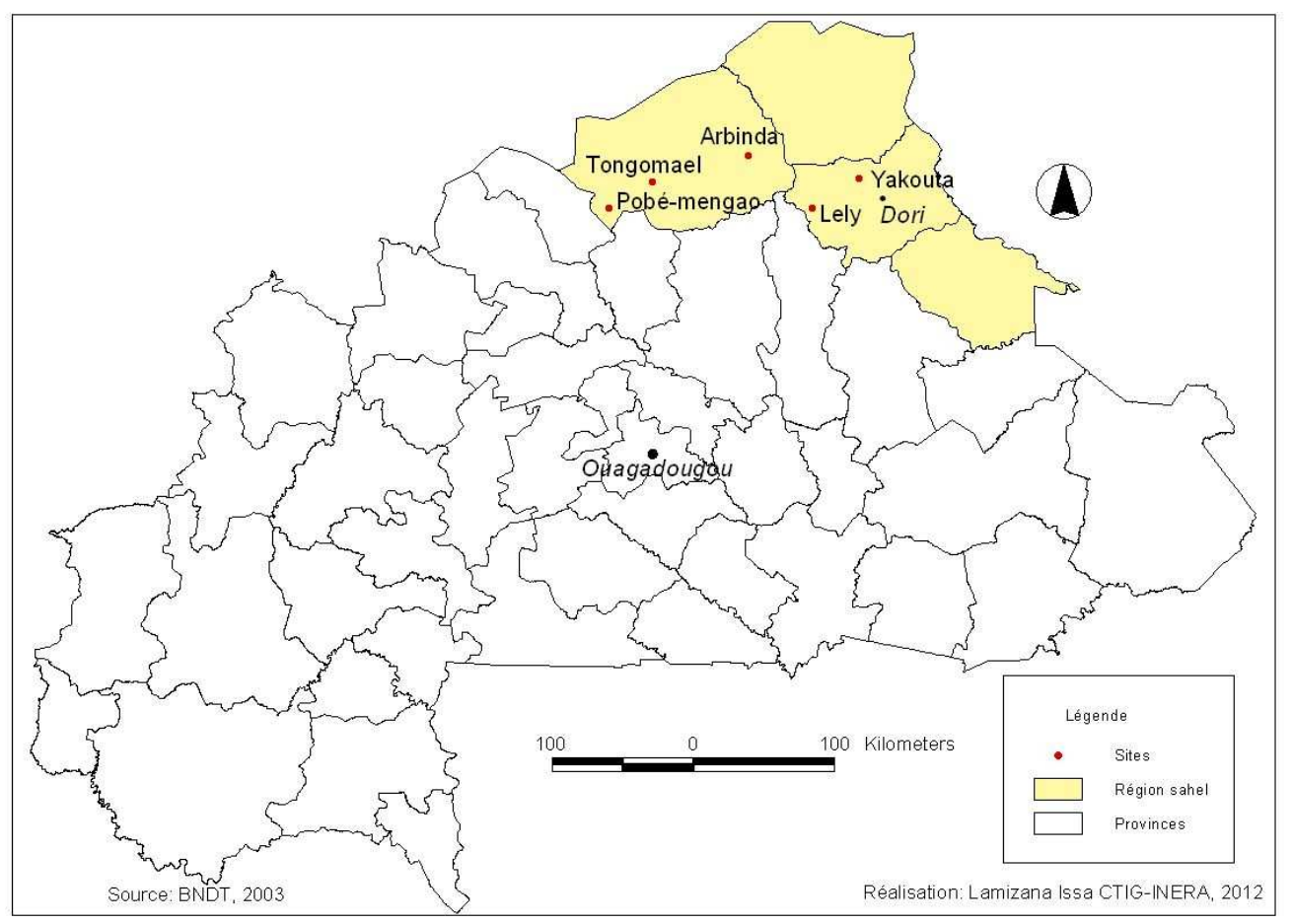

Figure 1: Carte de situation des cinq sites d'étude au Burkina Faso. 


\section{Analyse statistique des données}

L'analyse a consisté à une répartition des exploitations en quatre classes ou catégories de producteurs en fonction de leur niveau de ressource en bovins : [0 à 10 têtes], ]10 à 20 têtes], ]20 à 30 têtes] et > à 30 têtes (Tableau 1). En effet, cette espèce représente à elle seule près de $80 \%$ des UBT des herbivores de cette région (MRA, 2011). Cette répartition part de l'hypothèse que les producteurs produisent et stockent du fourrage en fonction $\mathrm{du}$ nombre d'animaux en présence, c'est-à-dire de leurs besoins. Les données d'enquête relatives aux ressources en bovins, aux petits ruminants et à la dotation en main d'œuvre familiale ont ensuite été soumises à une analyse de variance (ANOVA) à un critère de classification afin de tester la différence des classes. Le test de Scheffe (1959) a été utilisé pour identifier les moyennes qui diffèrent significativement au seuil de $5 \%$.

\section{RÉSULTATS}

\section{Caractéristiques générales des exploitations Dotation en ressources}

La dotation des ressources des ménages est indiquée au Tableau 2. Il apparaît selon la classification que la taille du ménage, les ressources en petits ruminants diffèrent significativement $(\mathrm{P}<0,05)$ selon le niveau de dotation des ménages en bovins. Les grands propriétaires de bovins sont également ceux qui disposent des plus grands effectifs en main d'œuvre et en petits ruminants. Le nombre d'ovins triple alors que celui des caprins quintuple lorsqu'on passe de la classe 1 à la 4 .

\section{Caractéristiques des producteurs enquêtés}

Des producteurs enquêtés, il ressort que les exploitations dont les chefs de ménage ayant un âge supérieur à 40 ans ont été les plus nombreuses dans toutes les classes de 1 à 4 (Tableau 3).

Les groupes ethniques ayant constitué l'échantillon d'enquête se composent essentiellement des Peul (31,6\%), des Fulcé (24,3\%), des Rimaïbé $(21,7 \%)$, de Mossi (19,5\%). L'importance des groupes ethniques varie en fonction des catégories ou classes de producteurs et on se rend compte que les mossis se concentrent dans la catégorie 2, les peulhs dans la 4, les Rimaïbé dans la 1 et les Fulcé dans les trois premières classes.

La répartition des producteurs en fonction de la taille du ménage montre que la plupart des exploitations enquêtées comptent plus de dix (10) personnes pour les classes 2 , 3 et 4 , tandis que la classe 1 regroupe surtout celles dont la taille est comprise entre 5 et 10 personnes. Le niveau d'instruction est généralement faible avec une moyenne de $5,5 \%$ pour la scolarisation et $8,8 \%$ pour l'alphabétisation. La classe 1 est celle qui comprend le plus de chefs d'exploitations d'instruits.

Le type d'activité dominante par ménage enquêté a été déterminé par catégorie. Les résultats montrent que les producteurs sont tous des agro pasteurs. L'agriculture demeure pour toutes les trois (3) premières classes, l'activité dominante pour 66 à $87 \%$ des producteurs. Dans la classe 4, l'élevage constitue la principale activité de $54,5 \%$ de producteurs. De même que dans la classe 2, l'élevage et l'agriculture constituent pour près de 40,6\% des producteurs, deux activités co dominantes.

En termes de collecte et conservation du fourrage, les enquêtes révèlent que $61,8 \%$ de producteurs pratiquent la fauche et conservation $\mathrm{du}$ fourrage, tandis que la collecte et conservation des résidus de récolte concernent en moyenne $88,2 \%$ de producteurs. Les plus fortes proportions de producteurs ne stockant pas de fourrage se trouvent dans les classes à faible niveau de ressources. Ainsi, peu de producteurs de la classe 1 pratiquent le stockage de fourrage comparé à ceux des autres classes. 
Évaluation quantitative et qualitative des fourrages stockés

Évaluation quantitative

Les stocks fourragers varient en fonction des classes d'exploitation. L'évaluation des stocks chez les producteurs pratiquant la collecte et la conservation a montré qu'en moyenne les résidus de récoltes $(85,4 \%)$ constituent l'essentiel du fourrage stocké. Les pailles naturelles $(5,8 \%)$, les fanes naturelles $(6,8 \%)$ et cultivées $(12,8 \%)$ sont en quantité moins importante que les tiges de céréales $(72,6 \%)$. Les cultures fourragères $(1,1 \%)$ et les fourrages ligneux $(0,8 \%)$ constituent des stocks marginaux. Les quantités de fourrage stockées sont en moyenne de 2042,7 kg/producteur (Tableau 4).

\section{Évaluation qualitative}

L'analyse bromatologique d'échantillons composites des fourrages stockés chez les producteurs a donné les résultats indiqués dans le Tableau 5. Les tiges de céréales sont de qualités médiocres, les fanes de légumineuses de bonnes qualités et les fourrages ligneux d'excellentes qualités fourragères.

De façon spécifique, le foin d'Alysicarpus ovalifolius (S. et T.) Léo., de Dactyloctenium aegyptium (L.) Willd., les fanes de niébé et d'arachide sont bien conservées. Les fanes de Zornia glochidiata Reichb. ex DC. par contre, sont généralement fauchées au cours de l'année à une période où elles ont perdu les feuilles, ce qui fait que la qualité fourragère est faible par rapport aux autres types de légumineuses conservées.

Les stocks de feuilles et gousses de Pterocarpus lucens Lepr. ex Guill. et Perr. et Acacia raddiana Savi sont riches en azote et en énergie. La paille de céréale (sorgho, mil) et celle des graminées (Schoenefeldia gracilis Kunth, Dactyloctenium aegyptium (L.) Willd.) ont les teneurs les faibles en azote. La richesse en énergie est cependant supérieure à 0,5 UF / kg de MS.

\section{Cultures fourragères}

La culture fourragère est faiblement pratiquée par rapport à la collecte et conservation des autres types de fourrages (résidus culturaux, fourrage naturel). En effet, $8,5 \%$ seulement sur les 205 producteurs enquêtés la pratiquent. De plus elle concerne surtout les producteurs des classes 1 et 2 . Les espèces mises en culture par les producteurs concernent essentiellement cinq espèces que sont le niébé fourrager $(25 \%)$, le sorgho fourrager $(25 \%)$, l'arachide fourragère $(12,5 \%)$ et la Dolichos lablab L. pour 37,5\%. Les principales raisons de cette faiblesse de la pratique des cultures fourragères sont très variables selon les producteurs. D'une façon générale, les problèmes de semences sont les plus importants chez plus de $46,4 \%$. A cela s'ajoute la méconnaissance des techniques des cultures fourragères $(35,9 \%)$. Les problèmes liés aux moyens financiers $(3,3 \%)$, manque de temps $(5,9 \%)$, insuffisance des parcelles de cultures $(3,3 \%)$ sont également évoqués par quelques producteurs (Tableau 6).

\section{Sites de collecte du fourrage}

La collecte du fourrage se fait sur différents types d'unités de végétation que sont les champs, les pâturages naturels de glacis, de bas - fonds, etc. en fonction de leurs potentialités fourragères. Les champs constituent les sites sur lesquels les producteurs collectent les résidus de récolte et beaucoup d'autres espèces telles Alysicarpus ovalifolius (S. et T.) Léo (48,5\%), Schoenefeldia gracilis Kunth (29,1\%), Zornia glochidiata Reichb. ex DC. (26,4\%). Ces espèces naturelles sont bien conservées dans les espaces inter - champs et se prêtent à la fauche pour la conservation. Les champs fournissant le fourrage naturel sont pour la plupart sur sites sableux et sur bas fonds. Les parcours naturels sur bas fonds et glacis 
regorgent surtout de réserves fourragères en termes de gousses d'Acacia raddiana Savi, de Piliostigma reticulatum (DC.) Hochst., de feuilles de Pterocarpus lucens Lepr. ex Guill. et Perr., de pailles naturelles (Schoenefeldia gracilis Kunth, Panicum laetum Kunth, Dactyloctenium aegyptium (L.) Willd., etc.). Les bas fonds sont riches en diverses autres espèces fauchées et stockées $(83,4 \%)$ (Tableau 7).

\section{Constitution des stocks de fourrage}

Les périodes de collecte dans l'année varient en fonction des producteurs mais aussi et surtout des catégories de fourrage. Si les résidus de récolte ne se stockent généralement qu'en période post-récolte $(97,8 \%)$, la collecte du fourrage naturel s'étend à toutes les saisons avec toutefois des pics en période des pluies $(46,8 \%)$ et post récolte $(42,1 \%)$. Les stocks de fourrages naturels constitués en saison sèche concernent surtout les gousses et feuilles d'espèces ligneuses. Les travaux de collecte et conservation des résidus de récoltes interviennent immédiatement après les récoltes. Cela permet aux agriculteurs d'assurer une meilleure gestion de leurs résidus de récolte et d'éviter l'exploitation par des troupeaux étrangers (Figure 2).

\section{Achat et vente des fourrages}

Les résultats au Tableau 8 montrent qu'en matière de vente et d'achat de fourrage il y a des différences entre producteurs en fonction de leur catégorie. Les producteurs à plus faible ressources sont ceux qui vendent le fourrage alors que ceux des autres classes sont plutôt acheteurs. En moyenne, seulement 9,3\% des producteurs enquêtés dans les villages effectuent des achats de fourrage et $26 \%$ des ventes. Les coûts d'achat et de revenus de vente du fourrage sont très variables entre producteurs. Pour les achats et les ventes ils sont respectivement en moyenne de 8681,6 et $24362,8 \mathrm{FCFA} /$ an/exploitation enquêtée. Les plus grands vendeurs se trouvent dans les classes 1 et 2 tandis que les acheteurs se trouvent dans les classes 3 et 4 .

\section{Gestion des stocks fourragers}

Il existe une certaine gestion des ressources fourragères collectées et stockées. En général, leur utilisation est régie par la disponibilité du fourrage sur les parcours. Les investigations montrent que les saisons froides et chaudes sont celles durant lesquelles le fourrage stocké est le plus utilisé. Cette importance varie en fonction du type de fourrage et de la catégorie de producteur. Il ressort que $83,7 \%$ des graminées, sont utilisées en saison sèche chaude contre $42,9 \%$ pour les stocks de ligneux fourrager. Les fanes sont surtout utilisées en période froide et chaude à raison respectivement de plus de $28,8 \%$ et $61,8 \%$.

Les producteurs moins nantis en ressources utilisent plus rapidement leurs stocks de fanes et fourrage ligneux que les classes riches chez lesquelles l'utilisation des stocks intervient surtout en période sèche chaude (Tableau 9).

Les stocks de fourrage destinés à l'affourragement des animaux sont judicieusement répartis en fonction des catégories d'animaux du troupeau. D'une façon générale, l'entretien des troupeaux occupe une place importante, particulièrement avec les pailles et foin de graminée $(90,9 \%)$ et le fourrage ligneux $(84,8 \%$ des stocks réservés). Les opérations d'embouche consomment près de $27,4 \%$ des stocks de fanes contre 9,2 et $15,2 \%$ respectivement pour les graminées et le fourrage ligneux en stocks. Par rapport à cette opération, les producteurs des petites classes utilisent plus leurs stocks que ceux des autres classes. 


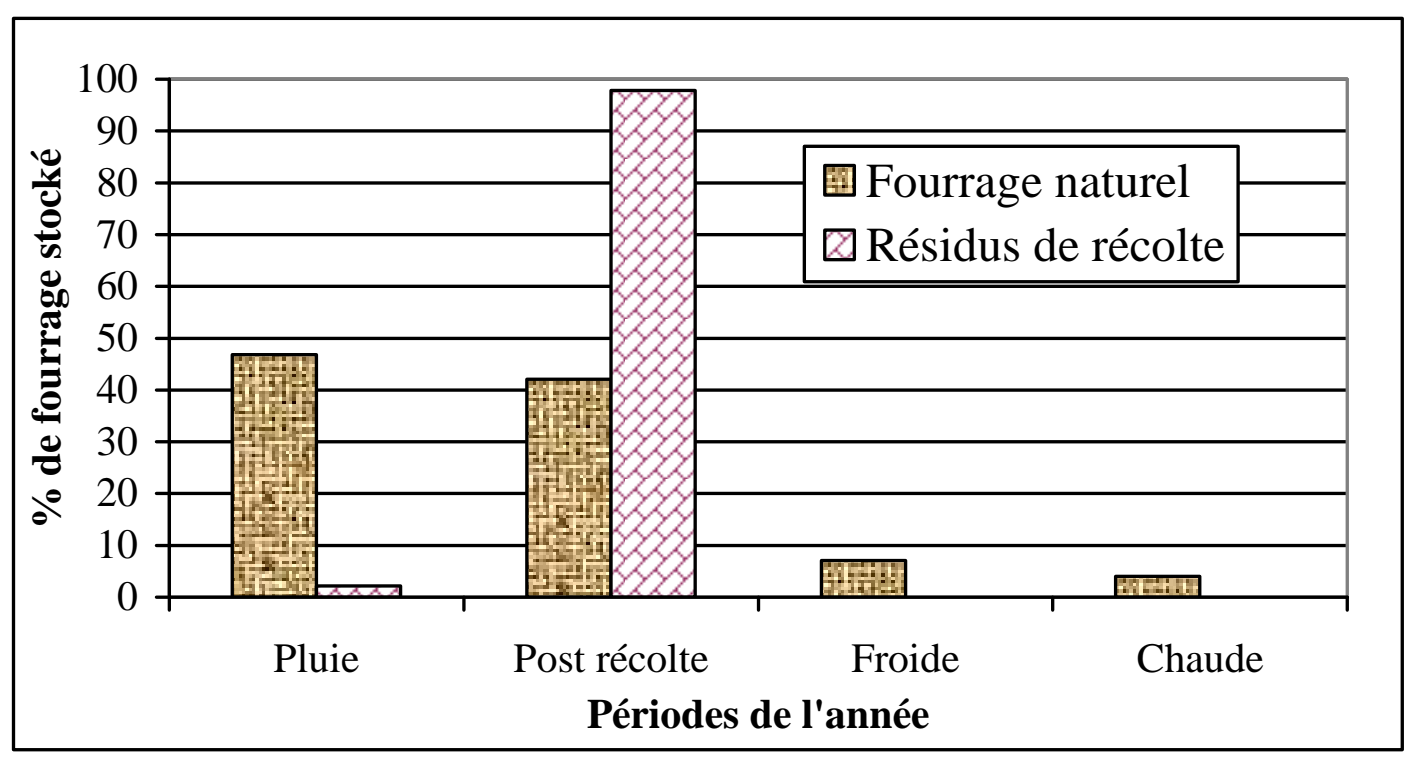

Figure 2: Dynamique de la constitution des stocks fourragers par saison.

Tableau 1: Répartition des exploitations enquêtées selon le niveau de dotation en bovins.

\begin{tabular}{|c|c|c|c|c|c|}
\hline \multirow[b]{2}{*}{ Localités } & \multicolumn{4}{|c|}{ Classes de bovins } & \multirow[b]{2}{*}{ Total } \\
\hline & [0 à 10 têtes] & ] 10 à 20 têtes] & ] 20 à 30 têtes] & > à 30 têtes & \\
\hline $\begin{array}{l}\text { Nombre } \\
\text { d'exploitation }\end{array}$ & 144 & 32 & 18 & 11 & 205 \\
\hline
\end{tabular}

Tableau 2: Dotation moyenne des classes d'exploitations en ressources animales et humaines.

\begin{tabular}{lcccc}
\hline Variables & \multicolumn{4}{c}{ Classes } \\
\cline { 2 - 5 } & $\mathbf{1}$ & $\mathbf{2}$ & $\mathbf{3}$ & $\mathbf{4}$ \\
\hline Taille des exploitations & $9,38^{\mathrm{a}}$ & $10,46^{\mathrm{b}}$ & $12,66^{\mathrm{b}}$ & $12,78^{\mathrm{ab}}$ \\
Effectifs bovins & $3,19^{\mathrm{a}}$ & $15^{\mathrm{b}}$ & $24,7^{\mathrm{c}}$ & $40,18^{\mathrm{d}}$ \\
Effectifs ovins & $9,59^{\mathrm{a}}$ & $18,67^{\mathrm{b}}$ & $24,06^{\mathrm{bc}}$ & $29,18^{\mathrm{c}}$ \\
Effectifs caprins & $9,38^{\mathrm{a}}$ & $19,06^{\mathrm{b}}$ & $20,41^{\mathrm{b}}$ & $49,01^{\mathrm{c}}$ \\
\hline
\end{tabular}

Sur la même ligne les chiffres suivis d'une même lettre ne sont pas significativement différents $(\mathrm{P}<0,05)$. 
A. KIEMA et al. / Int. J. Biol. Chem. Sci. 6(4): 1492-1505, 2012

Tableau 3: Caractéristiques des producteurs enquêtés (en \%).

\begin{tabular}{|c|c|c|c|c|c|c|}
\hline \multirow[t]{2}{*}{ Caractéristiques } & \multirow[t]{2}{*}{ Paramètres observés } & \multicolumn{4}{|c|}{ Classes de producteurs } & \multirow[t]{2}{*}{ Moyenne échantillon } \\
\hline & & $\mathbf{1}$ & 2 & 3 & 4 & \\
\hline \multirow[t]{4}{*}{ Ages en années } & $<20$ & 0,7 & & & & 0,2 \\
\hline & 20 à 30 & 16,7 & 12,5 & 5,6 & 18,2 & 13,2 \\
\hline & 30 à 40 & 29,9 & 21,9 & & 18,2 & 17,5 \\
\hline & $>40$ & 52,8 & 65,6 & 94,4 & 63,6 & 69,1 \\
\hline \multirow[t]{6}{*}{ Ethnies } & Peulh & 16,7 & 9,4 & 27,8 & 72,7 & 31,6 \\
\hline & Fulcé & 29,9 & 25 & 33,3 & 9,1 & 24,3 \\
\hline & Rimaïbé & 36,8 & 9,4 & 22,2 & 18,2 & 21,7 \\
\hline & Mossi & 11,1 & 50 & 16,7 & & 19,5 \\
\hline & Gourmantché & 3,5 & 3,1 & & & 1,7 \\
\hline & Autres & 2,1 & 3,1 & & & 1,3 \\
\hline \multirow[t]{3}{*}{ Structure / ménage } & $<5$ personnes & 35,4 & 15,6 & 22,2 & 36,4 & 27,4 \\
\hline & 5 à 10 personnes & 41,7 & 15,6 & & 18,2 & 18,9 \\
\hline & $>10$ personnes & 22,9 & 68,8 & 77,8 & 45,5 & 53,8 \\
\hline \multirow[t]{2}{*}{ Instruction } & Scolarisation & 15,6 & 2,4 & 2,9 & 1 & 5,5 \\
\hline & Alphabétisation & 23,9 & 3,4 & 4,9 & 2,9 & 8,8 \\
\hline \multirow[t]{3}{*}{ Activité principale } & Élevage & 5,6 & 3,1 & 22,2 & 54,5 & 21,4 \\
\hline & Agriculture & 86,8 & 56,3 & 66,7 & 45,5 & 63,8 \\
\hline & Agriculture/élevage & 7,6 & 40,6 & 11,1 & & 14,8 \\
\hline \multirow[t]{2}{*}{ Producteurs enquêtés } & Fauche de fourrage naturel & 63,2 & 50 & 72,2 & 60 & 61,8 \\
\hline & Collecte de résidus de récolte & 86,8 & 87,5 & 100 & 80 & 88,2 \\
\hline
\end{tabular}


Tableau 4: Quantité moyenne de fourrage stocké par exploitation (en kg de MS).

\begin{tabular}{|c|c|c|c|c|c|c|}
\hline \multirow[t]{2}{*}{ Types de fourrage } & \multicolumn{4}{|c|}{$\begin{array}{c}\text { Stocks de fourrage en } \mathrm{kg} / \text { Classes de } \\
\text { producteurs }\end{array}$} & \multicolumn{2}{|c|}{$\begin{array}{l}\text { Moyenne } \\
\text { échantillon }\end{array}$} \\
\hline & $\mathbf{1}$ & 2 & 3 & 4 & $\mathbf{K g}$ & $\%$ \\
\hline Paille de graminées naturelles & 77 & 70,5 & 213,1 & 116,9 & 119,4 & 5,8 \\
\hline Fanes de légumineuses naturelles & 107,5 & 248,5 & 93,6 & 106,4 & 139 & 6,8 \\
\hline Feuilles et gousses de ligneux & 12,8 & 10,1 & 21,5 & 20,2 & 16,2 & 0,8 \\
\hline Tiges de céréales & 1038,2 & 1685,6 & 1731,3 & 1475,7 & 1482,7 & 72,6 \\
\hline Fanes de légumineuses & 297,1 & 365 & 299 & 86,2 & 261,8 & 12,8 \\
\hline Autres types de fourrage & 2,3 & & & & 0,6 & 0,03 \\
\hline Cultures fourragères & 13,8 & 2,2 & 25,4 & 50,8 & 23,1 & 1,1 \\
\hline Stock fourrager total & 1548,7 & 2381,9 & 2383,9 & 1856,2 & 2042,7 & 100 \\
\hline
\end{tabular}

Tableau 5: Composition chimique des principaux types de fourrages stockés entre décembre et février.

\begin{tabular}{|c|c|c|c|c|c|c|c|c|}
\hline Type de fourrage & $\begin{array}{c}\text { Stock / } \\
\text { producteur } \\
\text { en kg }\end{array}$ & $\% \mathrm{MM}$ & $\begin{array}{l}\% \\
\text { CB }\end{array}$ & $\begin{array}{c}\% \\
\text { MAT }\end{array}$ & $\begin{array}{l}\text { UF / } \\
\text { kg } \\
\text { MS }\end{array}$ & $\begin{array}{c}\text { Mad } \\
(\mathrm{g} / \mathrm{kg} \\
\mathrm{de} \\
\mathrm{MS})\end{array}$ & $\begin{array}{l}\text { Mad } \\
\text { / UF }\end{array}$ & Catégorie \\
\hline $\begin{array}{l}\text { Alysicarpus ovalifolius } \\
\text { (S. et T.) Léo. } \\
\text { Zornia glochidiata }\end{array}$ & 125,1 & 7,05 & 28,85 & 9,14 & 0,76 & 49,71 & 90,53 & Bon \\
\hline Reichb. ex DC. & 13,8 & 5,63 & 38,69 & 4,35 & 0,54 & 5,21 & 10,76 & Pauvre \\
\hline Fanes d'arachide & 73,8 & 6,14 & 26,39 & 8,47 & 0,83 & 43,44 & 52,65 & Bon \\
\hline Fanes de niébé & 186,3 & 4,25 & 23,12 & 10,56 & 0,93 & 62,90 & 67,56 & Bon \\
\hline $\begin{array}{l}\text { Fanes de voandzou } \\
\text { Pterocarpus lucens }\end{array}$ & 1,7 & 4,55 & 9,44 & 5,9 & 1,07 & 19,6 & 18,4 & Pauvre \\
\hline $\begin{array}{l}\text { Lepr. ex Guill. et Perr. } \\
\text { Piliostigma } \\
\text { reticulatum (DC.) }\end{array}$ & 15,6 & 6,29 & 16,57 & 16,37 & 1,04 & 116,88 & 112,71 & Excellent \\
\hline $\begin{array}{l}\text { Hochst. } \\
\text { Dactyloctenium }\end{array}$ & 0,6 & 6,32 & 26,22 & 6,57 & 0,85 & 25,84 & 30,36 & Pauvre \\
\hline $\begin{array}{l}\text { aegyptium (L.) Willd. } \\
\text { Schoenefeldia gracilis }\end{array}$ & 15,6 & 6,51 & 25,37 & 8,74 & 0,84 & 45,99 & 54,89 & Bon \\
\hline $\begin{array}{l}\text { Kunth } \\
\text { Pennisetum }\end{array}$ & 30 & 4,33 & 34,56 & 6,69 & 0,63 & 26,95 & 43,79 & Pauvre \\
\hline $\begin{array}{l}\text { pedicellatum Trin. } \\
\text { Paille naturelle } \\
\text { (mélange) }\end{array}$ & 32,9 & 5,10 & 39,12 & 4,86 & 0,53 & 9,93 & 18,58 & Médiocre \\
\hline Andropogon gayanus & & & & & & & & \\
\hline Kunth & 8,1 & 4,56 & 42,99 & 5,28 & 0,45 & 13,80 & 30,54 & Pauvre \\
\hline Tiges de sorgho & 848,3 & 6,31 & 30,65 & 4,02 & 0,73 & 2,15 & 3,16 & Médiocre \\
\hline Tiges de mil & 634,4 & 5,37 & 48,21 & 4,51 & 0,45 & 6,70 & 14,82 & Médiocre \\
\hline Cultures fourragères & 23,1 & - & - & - & - & - & - & - \\
\hline $\begin{array}{l}\text { Diverses autres } \\
\text { espèces }\end{array}$ & 0,6 & 11,9 & 31,2 & 8,91 & 0,64 & 47,57 & 74,57 & Bon \\
\hline
\end{tabular}


Tableau 6: Paramètres influençant par producteur, la pratique de la culture fourragère (valeur en $\%)$.

\begin{tabular}{|c|c|c|c|c|c|c|}
\hline \multirow[t]{2}{*}{ Caractéristiques } & \multirow[t]{2}{*}{ Paramètres observés } & \multicolumn{4}{|c|}{ Classes de producteurs } & \multirow[t]{2}{*}{ Total } \\
\hline & & $\mathbf{1}$ & 2 & 3 & 4 & \\
\hline Niveau de pratique & Culture fourragère & 10 & 8,4 & 1 & & 8,5 \\
\hline \multirow[t]{4}{*}{ Espèces spéculées } & Niébé fourrager & 40 & & & & 25 \\
\hline & Arachide fourragère & & 50 & & & 12,5 \\
\hline & Dolichos lablab L. (dolique) & 20 & 50 & 100 & & 37,5 \\
\hline & Sorgho fourrager & 40 & & & & 25 \\
\hline \multirow{8}{*}{$\begin{array}{l}\text { Difficultés liées à la } \\
\text { pratique de la culture } \\
\text { fourragère }\end{array}$} & Manque de semences & 46,8 & 52,4 & 35,7 & 44,4 & 46,4 \\
\hline & Insuffisance de moyens financiers & 5,5 & & 7,1 & & 4,6 \\
\hline & Insuffisance de parcelles adéquates & 3,7 & & 7,1 & & 3,3 \\
\hline & Effectifs d'animaux insuffisants & & 4,8 & & & 0,7 \\
\hline & Manque de temps & 8,3 & & & & 5,9 \\
\hline & Culture difficile & & 4,8 & & & 0,7 \\
\hline & Ne connaît pas la technique & 33,9 & 33,3 & 42,9 & 55,6 & 35,9 \\
\hline & Manque d'appui technique & 1,8 & 4,8 & 7,1 & & 2,6 \\
\hline
\end{tabular}

Tableau 7: Contribution relative des unités de pâturage à la collecte du fourrage en \%.

\begin{tabular}{lcccc}
\hline Types de fourrage & \multicolumn{4}{c}{ Unités de pâturage } \\
\cline { 2 - 5 } & Champs & Glacis & Bas fond & Autres \\
\hline Paille naturelle (mélange) & 33,3 & 24,6 & 38,5 & 3 \\
Pterocarpus lucens Lepr. ex Guill. et Perr. & 16 & 61,9 & 20,1 & 2 \\
Pennisetum pedicellatum Trin. & 27 & 12,2 & 58,1 & 3 \\
Dactyloctenium aegyptium (L.) Willd. & 26,4 & 11,8 & 61,8 & \\
Acacia raddiana Savi. & & 100 & & \\
Alysicarpus ovalifolius (S. et T.) Léo. & 48,5 & 13 & 36,9 & 1 \\
Zornia glochidiata Reichb. ex DC. & 26,4 & 59,7 & 13,9 & \\
Panicum laetum Kunth & 46,7 & 36,7 & 16,7 & \\
Schoenefeldia gracilis Kunth & 29,1 & 57,3 & 13,6 & \\
Piliostigma reticulatum (DC.) Hochst. & 8,3 & 70,8 & 20,8 & \\
Autres espèces naturelles & & 16,7 & 83,4 & \\
Résidus de récolte & 100 & & & \\
\hline
\end{tabular}


Tableau 8: Montant annuel (FCFA/producteur) des échanges et taux d'utilisation (\%) des recettes de vente de fourrage.

\begin{tabular}{|c|c|c|c|c|c|c|}
\hline & \multirow[t]{2}{*}{ Paramètres } & \multicolumn{4}{|c|}{ Classes de producteurs } & \multirow[t]{2}{*}{ Total } \\
\hline & & $\mathbf{1}$ & 2 & 3 & 4 & \\
\hline \multirow[t]{2}{*}{ Vente } & Fourrage naturel $\%$ de producteurs & 18,1 & 6,3 & 5,6 & & 14,2 \\
\hline & Résidus de récolte $\%$ de producteurs & 16 & 0 & 5,6 & & 11,8 \\
\hline Achat & Achat en $\%$ de producteurs & 7,6 & 6,3 & 22,2 & 18,2 & 9,3 \\
\hline \multirow{4}{*}{$\begin{array}{l}\text { Vente de } \\
\text { fourrage }\end{array}$} & Moyenne fourrage naturel & 12359,6 & 52000 & 8000 & & 14943 \\
\hline & Moyenne résidus récolte & 9698,9 & & 3000 & & 9419,8 \\
\hline & Ecartype fourrage naturel & 9426,4 & 67882,3 & & & 18706 \\
\hline & Ecartype résidus récolte & 5405,8 & & & & 5461 \\
\hline \multirow{2}{*}{$\begin{array}{l}\text { Achat de } \\
\text { fourrage }\end{array}$} & Moyenne & 6790,9 & 3500 & 16688 & 8250 & 8681,6 \\
\hline & Ecartype & 4869,1 & 2121,3 & 9775 & 9546 & 7337,7 \\
\hline \multirow{6}{*}{$\begin{array}{l}\text { Utilisation des } \\
\text { revenues de } \\
\text { vente du } \\
\text { fourrage en } \%\end{array}$} & Vivres & 37,1 & 25 & & & 35,3 \\
\hline & Scolarité & 8,1 & & & & 7,4 \\
\hline & Habillement & 25,8 & 50 & 50 & & 27,9 \\
\hline & Constitution de troupeau & 24,2 & 25 & & & 23,5 \\
\hline & Santé & 4,8 & & 50 & & 5,9 \\
\hline & Autres & 3,2 & & & & 2,9 \\
\hline
\end{tabular}

Tableau 9: Gestion des stocks fourragers en fonction des périodes d'utilisation et des catégories d'animaux (valeurs en \%).

\begin{tabular}{llccccc}
\hline Type de fourrage & Périodes d'utilisation/ & \multicolumn{4}{c}{ Classes de producteurs } & Moyenne \\
\cline { 3 - 5 } & catégorie de troupeau & $\mathbf{1}$ & $\mathbf{2}$ & $\mathbf{3}$ & $\mathbf{4}$ & échantillon \\
\hline Graminées & Pluies & 1,8 & 3,2 & 3,6 & & 2,2 \\
& Post récolte & 3 & 4,4 & 3,6 & & 2,7 \\
& Froide & 17,5 & 13,9 & 14,4 & & 11,5 \\
& Chaude & 77,7 & 78,6 & 78,4 & 100 & 83,7 \\
Légumineuses & Pluies & 3,9 & 4,8 & 8,7 & & 4,4 \\
& Post récolte & 6,7 & 4,8 & 8,7 & & 5,1 \\
& Froide & 38,2 & 42,3 & 34,8 & & 28,8 \\
Feuilles/gousses de & Chaude & 51,2 & 48,1 & 47,8 & 100 & 61,8 \\
ligneux & Pluies & 0,8 & & & & 0,2 \\
& Post récolte & 4,6 & 8,4 & & & 3,2 \\
& Froide & 79,6 & 75,1 & 60 & & 53,7 \\
Utilisation des & Chaude & 15 & 16,7 & 40 & 100 & 42,9 \\
graminées & Troupeau entier & 90,6 & 83,3 & 89,5 & 100 & 90,9 \\
Utilisation des & Embouche & 9,4 & 16,7 & 10,5 & & 9,2 \\
légumineuses & Troupeau entier & 64 & 44,7 & 81,9 & 100 & 72,6 \\
Utilisation des & Embouche & 35,9 & 55,4 & 18,2 & & 27,4 \\
ligneux & Troupeau entier & 82,1 & 57,1 & 100 & 100 & 84,8 \\
\hline & Embouche & 18 & 42,9 & & & 15,2 \\
\hline
\end{tabular}




\section{DISCUSSION}

La collecte et la conservation du fourrage est une pratique courante de la majorité des agro pasteurs. Le nombre d'animaux est un critère déterminant du niveau d'activité ainsi que de la gestion des stocks de fourrages constitués. L'analyse des stratégies d'exploitation s'est donc appesantie sur ces facteurs les plus susceptibles d'influencer cette activité dans les localités du Sahel partageant les mêmes caractéristiques écologiques.

Les stocks fourragers concernent des espèces très variées dans différentes familles végétales. Les quantités de fourrage les plus stockées concernent les tiges de céréales (près de $80 \%)$, les fanes de légumineuses $(12,8 \%)$, et de fourrage naturel $(12,6 \%)$. Cependant, la nature des fourrages constituant les stocks peut être variable en fonction de la pluviosité qui détermine la qualité et les volumes d'eau disponibles sur les pâturages. Les années de mauvaises pluviosités conduisent les producteurs à effectuer plus de stocks qu'en années moins abondantes (Vokouma, 1998; Kiema, 2002). La main d'œuvre, la disponibilité du matériel et les ressources animales sont également des facteurs qui déterminent la collecte et la conservation du fourrage comme l'ont fait observé Bougma et al. (2001). Pour les grands propriétaires de troupeau, l'option de la transhumance est le plus souvent envisagée par rapport à la collecte et conservation du fourrage au regard des besoins en investissement de main d'œuvre, de moyen et de temps. Ainsi, une amélioration des conditions de travail chez les producteurs à plus faible niveau en ressources animales est susceptible de permettre un meilleur accroissement des activités de fauche et conservation du fourrage.

L'analyse bromatologique de ces fourrages confirme que les stocks fourragers de légumineuses et de ligneux sont de meilleures qualités en raison de leur teneur élevée en matière azotée et en énergie comme déjà indiqué par plusieurs auteurs (Piot et al.,
1980; Le Houérou, 1980). Les faibles teneurs enregistrées chez les graminées montrent que ce type de fourrage est plutôt stocké sous forme de paille que de foin, ce qui ne permet pas de satisfaire au besoin nutritionnel des animaux (Demarquilly et al., 1980). D'un constat général, la diversité de la qualité des fourrages en stock indique la difficulté qu'il y a à pouvoir constituer des rations alimentaires selon les objectifs de production. Cette situation serait surtout due à la variation du fourrage venant de divers sites de pâturages comprenant différentes espèces. Ces espèces ont des cycles végétatifs différents ce qui ne permet pas d'optimiser leurs meilleures périodes d'exploitation. Les cultures fourragères sont faiblement pratiquées et les différents travaux de vulgarisation se sont toujours heurtés à plusieurs contraintes dont les principales sont entre autres la faible technicité des producteurs, le manque de semences, de main d'œuvre, de matériel, aux pratiques traditionnelles d'exploitation des ressources pastorales, etc. Une amélioration de la production de fourrage par la culture et/ou l'aménagement des espaces de fauche et de conservation de fourrage peuvent permettre une augmentation des stocks fourragers et leurs qualités nutritionnelles.

D'un autre constat, les stocks constitués servent soit à l'affourragement des animaux, soit à la vente. Leur utilisation s'effectue selon des stratégies qui répondent aux besoins de production des producteurs. Les ressources financières issues de la vente de fourrage, servent à l'achat de vivres et à la constitution de troupeaux. L'importance des échanges varie en fonction des catégories de producteurs. Ils sont en moyenne de 24363 FCFA/producteur. Bien que les résidus de récoltes soient plus importants que les fourrages naturels, ces derniers ont une contribution plus élevée (61\%) aux recettes de ventes annuelles du producteur. Toutefois, il est à noter que ces valeurs sont relativement inférieures à celles données par Pinde (2002) selon lesquelles, les revenus des ventes de 
fourrage rapporteraient annuellement aux producteurs 57600 FCFA; mais il existe de grandes variations interannuelles liées essentiellement à la variation pluviométrique. Par ailleurs, selon plusieurs auteurs (Thébaud, 1998 ; Vokouma, 1998 ; Bougouma et al., 2001 ; Kiema, 2002), la fauche des plantes fourragères est devenue depuis ces dix dernières années une pratique assez répandue qui intéresse près de 74 à $95 \%$ des exploitations de la région du Sahel.

Cependant, l'activité connaît des difficultés qui sont essentiellement liées au manque de moyens financiers pour l'acquisition d'outils adéquats, au temps de travail qu'elle nécessite, etc. Elle se caractérise également par une très grande faiblesse des stratégies d'exploitation et de gestion des espaces pastoraux et des ressources fourragères.

Cette étude suggère plusieurs recommandations. Il s'agit essentiellement (i) de faire en sorte que des efforts soient faits pour l'amélioration des cultures fourragères. En effet, très peu de producteurs $(8,5 \%)$ s'adonnent à cette pratique comme cela a également été précisé par Pindé (2002). L'amélioration de la pratique passe par la mise à disposition des producteurs de semences à des prix subventionnés, la promotion de certaines cultures fourragères de rente, l'intensification de la sensibilisation/ formation, etc. Cette promotion entraînerait une augmentation du gain des producteurs intéressés et pourrait avoir des effets d'entraînement sur une grande majorité. (ii) Intensifier la création des zones de pâture et de collecte du fourrage, et assurer une meilleure réglementation de l'activité de fauche et conservation dans les villages. Ces questions doivent être traitées en concertation dans les Conseil Villageois de Développement (CVD) lors de l'élaboration des plans et des règles internes de gestion des communes. Cela permettra de minimiser les conflits sur le foncier en matière d'exploitation des ressources fourragères. (iii) Imaginer des codes ou méthodes simples de suivis d'impact de la végétation naturelle sur la dynamique quantitative et qualitative du fourrage. Ces méthodes pourraient être inspirées des résultats d'études expérimentales telles que celles portant sur l'impact de la fauche sur la dynamique de la végétation (Kiema et al., 2008), celles-ci pouvant servir de base dans la conduite d'expériences au niveau village. En effet, si dans le domaine de la restauration des pâturages, de nombreuses techniques existent (Kiema et al., 2007), les techniques de gestion ou d'exploitation des pâturages sont par contre très peu expérimentées. Les interventions des structures techniques et de producteurs sont plutôt orientées vers des travaux d'aménagement avec très peu d'approches d'exploitation durable liées certainement au fait que ces approches de gestion sont difficiles à mettre en œuvre et demandent une meilleure organisation des producteurs.

\section{Conclusion}

Sur la base des ressources productives (nombre d'animaux) quatre classes de paysans ont été distinguées. En saison sèche, ces classes déploient des stratégies différentes en matière de gestion des stocks alimentaires. Les producteurs les moins nantis en ressources animales ont tendance à être des vendeurs nets de fourrages tandis que les plus nantis des acheteurs. Les principales contraintes aux activités d'exploitation du fourrage naturel sont surtout d'ordre économique mais aussi de gestion de l'espace.

\section{REFERENCES}

Boudet G. 1991. Manuel sur les Pâturages Tropicaux et les Cultures Fourragères (2ème édn). Ministère de la Coopération et du Développement : IEMVT, Collection Manuels et Précis d'Elevage: France.

Boutrais J. 2007. Crises écologiques et mobilités pastorales au Sahel : les Peuls 
du Dallol Bosso (Niger). Sécheresse, 18: 5-12.

Bougouma V, Nignan M, Kiema A. 2001. Contribution à l'Etude de la Commercialisation des Fourrages au Burkina Faso. INERA-DPA : Ouagadougou.

Claude J, Grouzis M, Milleville P. 1991. Un Espace Sahélien : la Mare d'Oursi (Burkina Faso). Éditions de l'ORSTOM : Paris.

Demarquilly C, Andrieu J, Sauvant D, Dulphy JP. 1980. Composition et valeur nutritive des aliments. In Alimentation des Ruminants ( $2^{\text {ème }}$ edn), Jarrige $\mathrm{R}$ (ed). INRA: Paris; 469-518.

Guinko S. 1984. Végétation de la HauteVolta. Thèse de Doctorat ès Science Naturelles, Université, Bordeaux III, p. 394.

Kiema A. 2002. Ressources pastorales et leurs modes d'exploitation dans deux terroirs sahéliens du Burkina Faso. Mémoire de DEA, Institut du Développement Rural/ Université Polytechnique de Bobo Dioulasso, Burkina Faso, p. 66.

Kiema A, Nianogo AJ, Kaboré-Zoungrana C. 2007. Étude bilan des aménagements anti-érosifs dans les pâturages en région sahélienne du Burkina Faso. Études et Recherches Sahéliennes, 13: 21-28.

Kiema A, Nianogo AJ, Nacro HB. 2008. Effets de la fauche sur la dynamique de la végétation herbacée des pâturages de dépression (thalweg). Revue Science et Technique, Série Science Naturelle et Agronomie, 30(1): 89-102.

Le Houérou HN. 1980. Composition chimique et valeur nutritive des fourrages ligneux en Afrique tropicale occidentale. In Les
Ligneux Fourragers en Afrique, État Actuel des Connaissances, Le Houérou HN (éd). CIPEA: Addis Abeba, Éthiopie; 259-296.

MRA. 2011. Deuxième Enquête Nationale sur les Effectifs du Cheptel (ENEC II). Projection des Résultats et Analyses (Tome II). Ouagadougou.

Pinde S. 2002. Caractérisation de la coupe, la conservation et la commercialisation du fourrage dans les provinces du Séno et du Yagha. Mémoire d'ingénieur d'élevage, Institut du Développement Rural/ Université Polytechnique de Bobo Dioulasso, Burkina Faso, p. 83.

Piot J, Nebout JP, Nanot R, Toutain B. 1980. Utilisation des Ligneux Sahéliens par les Herbivores Domestiques: Étude Quantitative de la Zone Sud de la Mare d'Oursi, Haute-Volta. GERDAT : Paris.

Sawadogo I, Devineau J-L, Fournier A. 2012. État des ressources pastorales dans une terre d'accueil et de transit des pasteurs transhumants : le terroir de Kotchari (sudest du Burkina Faso). La Terre et la Vie Revue d'Écologie, 67(2): 157-178.

Scheffe H. 1959. The Analysis of Variance. Wiley: New York.

Thébaud B. 1998. Étude sur l'Economie des Ménages dans les Régions de Gorgadji et de Dori-Ouest. MEE-SP/CONAGESE: Ouagadougou.

Vokouma JP. 1998. Enquêtes sur les activités de fauche et de conservation du fourrage dans les villages à système d'élevage agro pastoraux sahéliens : cas des villages de Lelly et Mansila. Mémoire de fin cycle, École nationale d'élevage et de santé animale (ENESA), Ouagadougou, p. 45. 